\title{
Identification of a novel gene fusion (BMX-ARHGAP) in gastric cardia adenocarcinoma
}

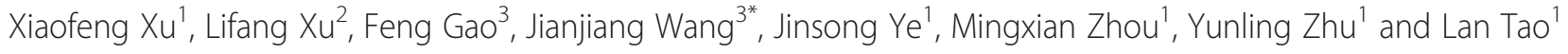

\begin{abstract}
Background: Gastric cardia adenocarcinoma (GCA) is one of the major causes of cancer related mortality worldwide. We aim to provide new understanding in the pathogenesis of GCA through investigations on gene expression alterations.

Methods: We preformed RNA-Seq for one pair of GCA and matched non-tumor tissues. Differentially expressed genes (DEGs) and fusion genes were acquired. PCR and gel analysis in additional 14 pairs of samples were performed to validate the chimeric transcripts.

Results: 1590 up-regulated and 709 down-regulated genes were detected. Functional analysis revealed that these DEGs were significantly overrepresented in gene ontology items of cell cycle, tumor invasion and proliferation. Moreover, we firstly discovered 3 fusion genes in GCA, including BMX-ARHGAP, LRP5- LITAF and CBX3-C15orf57. The chimeric transcript BMX-ARHGAP was validated and recurrently occurred in 4/15 independent tumor tissues.

Conclusions: Our results may provide new understanding of GCA and biomarkers for further therapeutic studies. Virtual Slides: The virtual slide(s) for this article can be found here: http://www.diagnosticpathology.diagnomx.eu/ vs/13000_2014_218
\end{abstract}

Keywords: Gastric cardia adenocarcinoma (GCA), RNA-Seq, Gene fusion

\section{Background}

Gastric cancer is the fourth most common malignant cancer and the second major cause of cancer-related death $[1,2]$. It is widely believed that gastric cancer is a heterogeneous disease with multiple environmental and genetic etiologies. To date, aberrant gene expression and epigenetic alterations were identified to be involved in the pathogenesis of gastric cancer. These abnormal changes could lead to perturbations in normal cellular homeostasis and neoplastic transformation of the gastric mucosa [3]. In particular, disruption in a number of regulatory signal pathways could create a permissive environment for carcinogenesis, invasiveness and metastasis. Gastric adenocarcinoma comprises $95 \%$ of the malignant gastric tumors [4]. It is classified as proximal (originating in the cardia) and distal (originating distal to the cardia). Different from distal adenocarcinomas, incidence of gastric

\footnotetext{
* Correspondence: wangjj9666@163.com

${ }^{3}$ Department of General Surgery, People s hospital, Jingjiang 214500, Jiangsu, China

Full list of author information is available at the end of the article
}

cardia adenocarcinoma (GCA) has increased significantly recently [5-7]. In addition, compared with distal adenocarcinomas, GCA seems to be more aggressive with deeper gastric wall invasion and worse prognosis [5,8]. Therefore, it is necessary to spend more efforts on the investigations for uncovering the pathogenesis of GCA.

With the rapid development of next generation sequencing (NGS), many cancer-related genes have been identified. NGS technology makes it possible to comprehensively illuminate whole map of genetic alteration of cancer. Specifically, massively parallel RNA-Sequencing (RNA-Seq) allows identification of entire gene expression and structural variation in individual samples, and facilitates fully characterization of cellular transcriptomes. Consequently, RNA-Seq becomes a revolutionary tool to study transcriptome profiling and measure the expression levels of various transcripts and isoforms [9]. Currently, investigations on GCA by using RNA-seq are still limited.

In this study, we generated comprehensive mRNA profiles in a pair of GCA and adjacent non-tumor tissues. We performed transcriptome-wide, unbiased analyses of the 
Table 1 Statistics of the sequenced reads and its mapping status

\begin{tabular}{|c|c|c|c|c|c|c|}
\hline Sample & Total reads & Mapped reads & Mapping ratio & Total base(bp) & Mapped base(bp) & Coverage() \\
\hline Non-tumor & 53611554 & 44218606 & $82.48 \%$ & 5361155400 & 4421860600 & 38.63 \\
\hline Tumor & 61217124 & 49477807 & $80.82 \%$ & 6121712400 & 4947780700 & 43.22 \\
\hline
\end{tabular}




RNA-Seq data to identify different kinds of gene transcriptional aberrations (mRNA expression and chimeric transcript) to investigate the molecular mechanism of gastric cancer pathogenesis. Interesting fusion genes were further validated in other independent samples. Our results may provide new understanding of GCA pathogenesis and new targets for future therapeutic studies.

\section{Methods}

\section{Sample information}

The GCA tissue and adjacent non-tumor tissue $(5 \mathrm{~cm}$ away from tumor) were collected from a 65 years old male patient who was diagnosed as T4N0M0 stage IIB GCA in 2012. The tumor size was $541 \mathrm{~cm}$. The tumor was moderately differentiated and didn $t$ spread to nearby lymph nodes or other organs. However, both venous invasion and nerve invasion were positive.

For validation of interesting fusion genes, another 14 pairs of stage IIB GCA and adjacent non-tumor tissues were obtained with the same procedure. Signed informed consent documents were obtained from all patients. The scientific use of these samples was approved by the Institutional Review Boards of the Peoples hospital, Jingiang, Jiangsu, China.

Table 2 Gene ontology items and pathways that significantly overrepresented with differentially expressed genes

\begin{tabular}{lll}
\hline ID & Description & $\boldsymbol{P}$ value \\
\hline Gene ontology & & \\
GO:0022403 & cell cycle phase & $2.11 \mathrm{E}-15$ \\
GO:0000279 & M phase & $4.11 \mathrm{E}-15$ \\
GO:0007049 & cell cycle & $7.68 \mathrm{E}-14$ \\
GO:0000280 & nuclear division & $1.87 \mathrm{E}-13$ \\
GO:0007067 & mitosis & $1.87 \mathrm{E}-13$ \\
GO:0048285 & organelle fission & $4.94 \mathrm{E}-13$ \\
GO:0022402 & cell cycle process & $5.06 \mathrm{E}-13$ \\
GO:0000087 & M phase of mitotic cell cycle & $5.06 \mathrm{E}-13$ \\
GO:0000278 & mitotic cell cycle & $6.84 \mathrm{E}-11$ \\
GO:0051301 & cell division & $4.74 \mathrm{E}-10$ \\
GO:0042127 & regulation of cell proliferation & $6.42 \mathrm{E}-07$ \\
GO:0007155 & cell adhesion & $1.47 \mathrm{E}-06$ \\
GO:0022610 & biological adhesion & $1.59 \mathrm{E}-06$ \\
GO:0007059 & chromosome segregation & $1.47 \mathrm{E}-05$ \\
GO:0007051 & spindle organization & $1.99 \mathrm{E}-05$ \\
GO:0007010 & cytoskeleton organization & $3.29 \mathrm{E}-05$ \\
KEGG pathway & & \\
hsa05130 & Pathogenic Escherichia coli infection & $9.84 \mathrm{E}-03$ \\
hsa04512 & ECM-receptor interaction & $1.29 \mathrm{E}-02$ \\
hsa04110 & Cell cycle & $1.86 \mathrm{E}-02$ \\
\hline
\end{tabular}

Table 3 The differentially alternative splicing events

\begin{tabular}{lllllll}
\hline & A3SS & A5SS & MXE & RI & SE & Total \\
\hline Tumor vs. Non-tumor (raw) & 31 & 38 & 53 & 75 & 114 & 311 \\
Tumor vs. Non-tumor (filtered) & 5 & 10 & 11 & 12 & 23 & 61 \\
\hline
\end{tabular}

RNA-Seq library preparation and Illumina sequencing Total RNA was isolated from frozen tissue by Trizol (Invotrogen) and its quality was assessed using Agilent Bioanalyzer. RNA-Seq libraries were prepared by TruSeq RNA Sample Prep Kit (Illumina Cat. No. FC-122-1001) according to standard protocols. Then the Libraries were sequenced on an Illumina Genome Analyzer IIx with 115 bp pair-end read length.

\section{RNA-Seq data processing}

For the raw data, low quality reads were filtered according to the criteria as follows: (1) reads containing adaptors were filtered; (2) nucleotides with a quality score lower than 20 were trimmed. The clean reads were aligned against both genome hg19 and transcripts reference using Bowtie 2.0.0 [10] and Tophat1.3.1 [11]. The mapping reads without biological interest, such as ribosomal RNAs and mitochondrial RNA were removed. The results of read mapping was visualized by using Integrative Genomics Viewer (IGV 2.0.26) [12].

\section{Detection of differentially expressed genes (DEGs)}

To identify DEGs, the values of human genes were normalized by per kilobase of exon per million mapped reads (FPKM) using Cufflinks (1.0.3) [13]. DEGs between GCA and non-tumor tissues were determined with significance cutoff of q-value $<0.05$. In addition, gene ontology (GO) and KEGG pathway enrichment analyses were performed via the web tool DAVID (http://david.abcc.ncifcrf. gov/) [14] with the false discovery rate (FDR) $<0.05$.

\section{Alternative splicing event detection}

The alternative splicing events were detected by MISO software [15], including alternative 3 splice sites (A3SS), alternative 5 splice sites (A5SS), mutually exclusive exons (MXE), retained intron (RI) and skipped exons (SE). Differentially expressed isoforms were further identified with following criteria: 1) the value of gene expression difference $>0.2$; 2 ) Bayes factors $>10 ; 3$ ) inclusive reads $>1$,

Table 4 Summary of candidate fusion genes detected by RNA-Seq

\begin{tabular}{lllll}
\hline No. & Gene1 & Gene2 & Break point1 & Break point2 \\
\hline 1 & BMX & ARHGAP12 & chrX:15548096 & chr10:32217611 \\
2 & LRP5 & LITAF & chr11:68125315 & chr16:11650591 \\
3 & C15orf57 & CBX3 & chr15:40854974 & chr7:26241386 \\
\hline
\end{tabular}



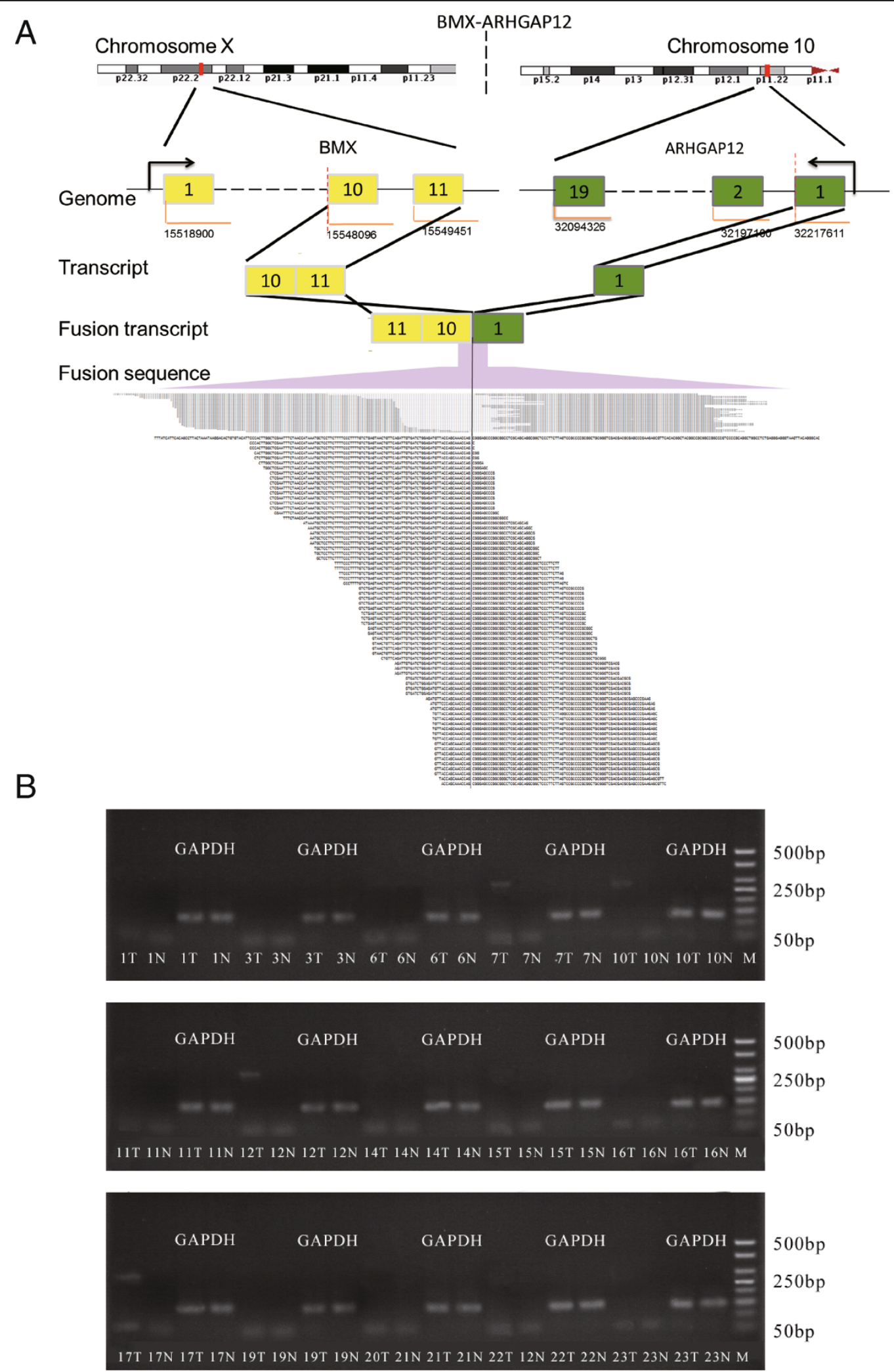

Figure 2 (See legend on next page.) 
(See figure on previous page.)

Figure 2 Detection and validation of the fusion gene BMX-ARHGAP in gastric cardia adenocarcinoma. A. BMX locates in chromosome $X$ and ARHGAP locates in chromosome 10. The breakpoint of BMX-ARHGAP is supported by both paired reads and single reads. B. Validation of the gene fusion with the target band of $260 \mathrm{bp}$. Sample 10 is the sequencing sample. T and N represent tumor and non-tumor tissue, respectively. It is obviously the expected target band is present in the tumor tissues of sample 7, 10, 12, and 17.

exclusive reads $>1$ and sum of inclusive reads and exclusive reads $>10$.

\section{Fusion gene identification}

Fusion gene was identified by Defuse [16] and TopHat [17]. The filtering processes of Defuse were carried out as previously described [18]. Fusion genes detected by TopHat should meet the following criteria: 1) support reads number should be more than $3 ; 2$ ) the supporting reads should not be mapped to ribosomal protein or small nuclear ribonucleoproteins. Only fusion genes detected by both methods were remained.

\section{Gene fusion validation}

To validate fusion transcripts, we performed PCR and gel analysis in the sequence sample and another 14 pairs of GCA and adjacent non-tumor tissues. All patients were also diagnosed as T4N0M0 stage IIB GCA. Primer pairs were designed using Primer 5 software, and RTPCR was performed using the following procedure: $94 \mathrm{C}$ for $1 \mathrm{~min}, 40$ cycles of $94 \mathrm{C}$ for $20 \mathrm{sec}, 55 \mathrm{C}$ for $20 \mathrm{sec}$ and $72 \mathrm{C}$ for $15 \mathrm{sec}$, followed by $72 \mathrm{C}$ for $1 \mathrm{~min}$. The PCR products of the fusion genes were visualized through agarose gel electrophoresis.

\section{Results}

In order to explore the spectrum of gene transcriptional changes in GCA, we sequenced the whole gene transcripts in one pair of GCA and matched non-tumor tissues from a 65 years old patient. We generated a total of 5.36 and $6.12 \mathrm{~Gb}$ data for the tumor and non-tumor tissue, respectively. Approximately $80 \%$ reads were mapped to the reference genome, achieving an average depth of coverage of $38.63 \mathrm{X}$ and $43.32 \mathrm{X}$, respectively (Table 1 ). The sequencing reads achieved fairly well evenness and integrity (Figure 1).

Next we analyzed gene expression level via calculating FPKM using the Cufflinks. About 15 thousand genes were expressed (FPKM $>1$ ) in our samples, among which 1590 genes were up-regulated and 709 genes were down-regulated in tumor. According to the enrichment analysis, these DEGs were significant overrepresented in 16 GO terms and 3 pathways, which were related to cell cycle, tumor invasion and proliferation (Table 2).

Alternative splicing events were also detected by using MISO. We found 311 differentially alternative spliced events. After the filtering process, 61 alternative splicing events were remained according to Bayes factors, Psi values and confidence intervals (Table 3 ).

Many fusion genes were reported as the potential cause for tumorigenesis, including gastric cancer $[19,20]$. Using Defuse and TopHat, 7 candidate fusion genes were identified with stringent filtering criteria. And finally 3 fusion genes were selected by TopHat realignment and manual inspection (Table 4, Figure 2). The fusion gene $B M X$ ARHGAP was validated using RT-PCR and gel analysis (Table 5), which was recurrently present in tumor tissues in about 26.7\% (4/15) GCA patients (Figure 2).

\section{Discussion}

The development of cancer is a multistep process during which cells acquires a series of mutations that eventually lead to unrestrained cell growth and division, inhibition of cell differentiation, and evasion of cell death. In order to comprehensively study aberrant gene expression in GCA, we performed the current study by using paired-end RNA-Seq technology. The results revealed the information of DEGs, alternative splicing, and gene fusion, which may have potential application in therapeutic studies.

In the present study, over $400 \mathrm{M}$ reads were sequenced on the Illumina platform, reaching about $40 \mathrm{X}$ coverage for the whole transcriptome. With those high sequencing depth, 2299 DEGs were detected between GCA and nontumor tissue. Further analysis showed ECM and cell cycle were the most enriched biological pathway among those abnormal expressed genes.

Alternative splicing is an important regulatory process during gene expression and substantially results in diverse transcripts. Abnormally spliced mRNAs were also found in multiple cancerous cells, including gastric cancer, breast cancer, and colon cancer [21,22]. In our study, 61 alternative spliced events were identified by using MISO software. Among them, $C D 44$ was also found be abnormally spliced in colorectal cancer and was suggested to be the character of metastatically potent tumor cells [23].

Table 5 The primer sequences used in PCR and gene fusion validation

\begin{tabular}{lll}
\hline Gene & Forward $\mathbf{( 5 - 3}$ ) & Reverse $\mathbf{~ 5 ~} \mathbf{- 3}$ ) \\
\hline GAPDH* & CATGAGAAGTATGACAACAGCCT & $\begin{array}{l}\text { AGTCCTTCCACG } \\
\text { ATACCAAAGT }\end{array}$ \\
${ }^{\$}$ BMX & GTGTACATTCCCACTTGGCTCG & \\
${ }^{\$}$ ARHGAP12 & & CCTGTAACTTAC \\
& & CCTCCCTCAG \\
\hline
\end{tabular}

*the control; ${ }^{5}$ primer used to amplify gene fusion. 
Gene fusion is another oncogenic activation mechanisms involved in the development of various types of malignancies including leukemia, lymphoma, breast and prostate cancer [24]. Importantly, several fusion genes, DUS4L $B C A P 29$ [19], CD44-SLC1A2 [20] have been reported in gastric cancer in western country. In our study, 3 chimeric transcripts were supported by the two detection methods (Table 4). Among them, CBX3-C15orf57 was detected in healthy human beings before [25]. In addition, both $C B X 3$ and $C 15$ orf57 were recurrently partnered with others in breast cancer [26]. Our results implicate the potential involvement of CBX3-C15orf57 in GCA. BMX-ARHGAP12 was firstly validated and was detected recurrently in 4 out of 15 GCA patients (Figure 2). BMX encodes a nonreceptor tyrosine kinase and ARHGAP12 encodes a Rho GTPase activating protein. It is possible that the chimeric transcript active the BMX mediation of tumorigenicity in GCA patients since BMX has been suggested to promote tumor growth and metastasis other cancers [27,28]. In addition, overexpression of ARHGAP12 would impair cell scattering, invasion and adhesion to fibronectin [29]. This fusion gene may play important roles in the tumorigenesis and may be potential targets for novel therapeutic strategy.

\section{Conclusions}

In summary, we performed transcriptome-wide analysis to identify gene expression aberrations in GCA. One of identified fusion gene, $B M X-A R H G A P 12$, was further confirmed in another independent patient. Our results may provide new understanding of the pathogenesis and new targets for further therapeutic investigations.

\section{Competing interests}

The authors declare that they have no competing interests.

\section{Authors contributions}

JW conceived of the study and revised the manuscript. XX and LX drafted the manuscript. FG performed statistical analysis. JY and MZ revised the manuscript. $Y Z$ and $L T$ performed validation experiments. All authors read and approved the final manuscript.

\section{Author details}

${ }^{1}$ Clinical Laboratory, People s hospital, Jingjiang 214500, Jiangsu, China. ${ }^{2}$ Department of Gastroenterology, People s hospital, Jingjiang 214500, Jiangsu, China. ${ }^{3}$ Department of General Surgery, People s hospital, Jingjiang 214500, Jiangsu, China.

Received: 2 July 2014 Accepted: 27 October 2014

Published online: 13 December 2014

\section{References}

1. Shen L, Shan Y-S, Hu H-M, Price TJ, Sirohi B, Yeh K-H, Yang Y-H, Sano T, Yang H-K, Zhang X, Park SR, Fujii M, Kang Y-K, Chen L-T: Management of gastric cancer in Asia: resource-stratified guidelines. Lancet Oncol 2013, 14:e535 e547.

2. Jemal A, Bray F, Center MM, Ferlay J, Ward E, Forman D: Global cancer statistics. CA Cancer I Clin 2011, 61:69 90

3. Nagini S: Carcinoma of the stomach: a review of epidemiology, pathogenesis, molecular genetics and chemoprevention. World J Gastrointest Oncol 2012, 4:156 169.
4. Dicken BJ, Bigam DL, Cass C, Mackey JR, Joy AA, Hamilton SM: Gastric adenocarcinoma: review and considerations for future directions. Ann Surg 2005, 241:27 39.

5. Brown LM, Devesa SS: Epidemiologic trends in esophageal and gastric cancer in the United States. Surg Oncol Clin N Am 2002, 11:235 256.

6. Orengo MA, Casella C, Fontana V, Filiberti R, Conio M, Rosso S, Tumino R, Crosignani P, De Lisi V, Falcini F, Vercelli M, Group AW: Trends in incidence rates of oesophagus and gastric cancer in Italy by subsite and histology, 1986 1997. Eur J Gastroenterol Hepatol 2006, 18:739 746.

7. Fock KM: Review article: the epidemiology and prevention of gastric cancer. Aliment Pharmacol Ther 2014, 40:250 260.

8. Piazuelo MB, Correa P: Gastric cancer: overview. Colombia Medica 2013, 44:192 201

9. Wang Z, Gerstein M, Snyder M: RNA-Seq: a revolutionary tool for transcriptomics. Nat Rev Genet 2009, 10:57 63.

10. Langmead B, Trapnell C, Pop M, Salzberg SL: Ultrafast and memory-efficient alignment of short DNA sequences to the human genome. Genome Biol 2009, 10:R25.

11. Kim D, Pertea G, Trapnell C, Pimentel H, Kelley R, Salzberg SL: TopHat2 accurate alignment of transcriptomes in the presence of insertions, deletions and gene fusions. Genome Biol 2013, 14:R36

12. Robinson JT, Thorvaldsdottir H, Winckler W, Guttman M, Lander ES, Getz G, Mesirov JP: Integrative genomics viewer. Nat Biotechnol 2011, 29:24 26.

13. Trapnell C, Roberts A, Goff L, Pertea G, Kim D, Kelley DR, Pimentel H, Salzberg SL, Rinn JL, Pachter L: Differential gene and transcript expression analysis of RNA-seq experiments with TopHat and Cufflinks. Nat Protoc 2012, 7:562 578

14. da Huang W, Sherman BT, Lempicki RA: Systematic and integrative analysis of large gene lists using DAVID bioinformatics resources. Nat Protoc 2009, 4:44 57

15. Katz Y, Wang ET, Airoldi EM, Burge CB: Analysis and design of RNA sequencing experiments for identifying isoform regulation. Nat Methods 2010, 7:1009 1015 .

16. McPherson A, Hormozdiari F, Zayed A, Giuliany R, Ha G, Sun MG, Griffith M, Heravi Moussavi A, Senz J, Melnyk N, Pacheco M, Marra MA, Hirst M, Nielsen TO, Sahinalp SC, Huntsman D, Shah SP: deFuse: an algorithm for gene fusion discovery in tumor RNA-Seq data. PLOS Comput Biol 2011, 7:e1001138.

17. Kim D, Salzberg SL: TopHat-Fusion: an algorithm for discovery of novel fusion transcripts. Genome Biol 2011, 12:R72

18. Steidl C, Shah SP, Woolcock BW, Rui L, Kawahara M, Farinha P, Johnson NA, Zhao Y, Telenius A, Neriah SB, McPherson A, Meissner B, Okoye UC, Diepstra A, van den Berg A, Sun M, Leung G, Jones SJ, Connors JM, Huntsman DG, Savage KJ, Rimsza LM, Horsman DE, Staudt LM, Steidl U, Marra MA, Gascoyne RD: MHC class II transactivator CIITA is a recurrent gene fusion partner in lymphoid cancers. Nature 2011, 471:377 381.

19. Kim HP, Cho GA, Han SW, Shin JY, Jeong EG, Song SH, Lee WC, Lee KH, Bang D, Seo JS, Kim Jl, Kim TY: Novel fusion transcripts in human gastric cancer revealed by transcriptome analysis. Oncogene 2013, doi:10.1038/ onc.2013.490.

20. Tao J, Deng NT, Ramnarayanan K, Huang B, Oh HK, Leong SH, Lim SS, Tan IB, Ooi CH, Wu J, Lee M, Zhang S, Rha SY, Chung HC, Smoot DT, Ashktorab H, Kon OL, Cacheux V, Yap C, Palanisamy N, Tan P: CD44-SLC1A2 gene fusions in gastric cancer. Sci Transl Med 2011, 3:77ra30.

21. Li H, Guo L, Li J, Liu N, Liu J: Alternative splicing of RHAMM gene in chinese gastric cancers and its in vitro regulation. Zhonghua Yi Xue Yi Chuan Xue Za Zhi 2000, 17:343 347.

22. Pal S, Gupta R, Davuluri RV: Alternative transcription and alternative splicing in cancer. Pharmacol Ther 2012, 136:283 294.

23. Banky B, Raso-Barnett L, Barbai T, Timar J, Becsagh P, Raso E: Characteristics of CD44 alternative splice pattern in the course of human colorectal adenocarcinoma progression. Mol Cancer 2012, 11:83.

24. Heim S, Mitelman F: Molecular screening for new fusion genes in cancer. Nat Genet 2008, 40:685 686.

25. Schrider DR, Navarro FC, Galante PA, Parmigiani RB, Camargo AA, Hahn MW, de Souza SJ: Gene copy-number polymorphism caused by retrotransposition in humans. PLoS Genet 2013, 9:e1003242.

26. Chen K, Navin NE, Wang Y, Schmidt HK, Wallis JW, Niu B, Fan X, Zhao H, McLellan MD, Hoadley KA, Mardis ER, Ley TJ, Perou CM, Wilson RK, Ding L: BreakTrans: uncovering the genomic architecture of gene fusions. Genome Biol 2013, 14:R87. 
27. Guo S, Sun F, Guo Z, Li W, Alfano A, Chen H, Magyar CE, Huang J, Chai TC, Qiu S, Qiu Y: Tyrosine kinase ETK/BMX is up-regulated in bladder cancer and predicts poor prognosis in patients with cystectomy. PLOS ONE 2011, 6:e17778.

28. Cenni B, Gutmann S, Gottar-Guillier M: BMX and its role in inflammation, cardiovascular disease, and cancer. Int Rev Immunol 2012, 31:166 173.

29. Gentile A, D Alessandro L, Lazzari L, Martinoglio B, Bertotti A, Mira A, Lanzetti L, Comoglio PM, Medico E: Met-driven invasive growth involves transcriptional regulation of Arhgap12. Oncogene 2008, 27:5590 5598.

doi:10.1186/s13000-014-0218-4

Cite this article as: Xu et al:: Identification of a novel gene fusion (BMX-

ARHGAP) in gastric cardia adenocarcinoma. Diagnostic Pathology 2014 9:218.

\section{Submit your next manuscript to BioMed Central and take full advantage of:}

$\otimes$ Convenient online submission

$\otimes$ Thorough peer review

$\otimes$ No space constraints or color $₫$ gure charges

$\otimes I m m e d i a t e$ publication on acceptance

\Inclusion in PubMed, CAS, Scopus and Google Scholar

$\otimes$ Research which is freely available for redistribution 